Journal of Medical and Health Studies (JMHS)

ISSN: 2710-1452

DOI: $10.32996 /$ jmhs

Journal Homepage: www.al-kindipublisher.com/index.php/jmhs

\title{
Characteristics of Malignant Pleural Effusion Patients from a Single Oncology Center
}

\author{
Sharif Othman ${ }^{1} \square$ Mohammad Al Khayyer $^{2}$ and Firas Hussein ${ }^{3}$ \\ ${ }^{1}$ Department of internal medicine, Tishreen University Hospital, Syria \\ ${ }^{2}$ Department of pulmonology, Tishreen University Hospital, Syria \\ ${ }^{3}$ Department of hematology, Tishreen University Hospital, Syria \\ $\square$ Corresponding Author: Sharif Othman, E-mail: sharifothman92@outlook.com
}

\section{ARTICLE INFORMATION}

Received: 08 October 2021

Accepted: 25 October 2021

Published: 04 November 2021

DOI: $10.32996 / j m h s .2021 .2 .2 .11$

\section{KEYWORDS}

Malignant pleural effusion, lung cancer, adenocarcinoma, pleurodesis

\section{ABSTRACT}

Malignant pleural effusion is defined as an effusion containing neoplastic cells. Despite the advances made, its treatments are palliative, with a low average survival rate. Prognosis varies depending on many factors. As a result of this difference, it is difficult to predict the prognosis. Therefore, following patients in terms of survival rates and their correlation with these factors enables us to draw results that serve future research related to diagnostic and therapeutic methods. This is an observational, prospective study on patients with malignant and semi-malignant pleural effusions from a single oncology center between January and June 2020. The patients' complete characteristics were recorded. Survival time was calculated from the diagnosis of pleural effusion until death or the passing of a year, whichever happened first. The final patient population was 89. We relied upon the IBM SPSS Statistics Version 20 program to calculate the statistical parameters and analyze the results. Men patients predominated our study and so did the patients younger than 60. Lung tumors were the most common cause. Histologically, adenocarcinoma occupied the largest percentage. The effect of adenocarcinoma on survival was statistically $(P<0.001)$ and clinically significant, greatly reducing these rates. Finally, pleurodesis was a statistically significant factor $(P=0.04)$ in improving survival. Malignant pleural effusion is associated with low survival rates. Lung tumors are the most common cause, while adenocarcinoma is the most common histological type, and it is of great statistical importance in reducing the survival rates. Finally, pleurodesis was associated with significantly better survival time.

\section{Introduction}

Malignant pleural effusion (MPE) is defined as a pleural effusion containing malignant cells (Rice et al., 2006). It is a common manifestation in patients with metastatic tumors, as it is seen in about 15 percent of patients with tumors (Henschke et al., 1991; Sahn, 1988). Lung and breast tumors, followed by lymphoma, gynecological tumors, and malignant mesothelioma are the most common causes (Anderson et al., 1974). The survival of patients with MPE after its diagnosis ranges between 3 and 12 months (Sahn, 1998). This effusion usually presents with dyspnea, cough, and chest pain. The severity of these symptoms is related to the amount (size) of the effusion and the condition of the patient's cardiovascular and respiratory systems (Sahn, 1988). Despite the advances in treatment methods for tumors, the treatments for MPE are still palliative, with a low average survival rate as mentioned above (Prakash \& Reiman, 1985). The prognosis for patients varies greatly and depends on a number of factors such as the primary tumor and its stage, the patient's clinical condition, the patient's age, pleural fluid proteins, and other factors (Asakura et al., 2005; Heffner et al., 2000). As a result of this disparity and due to the wide spectrum of tumors causing malignant effusions; it is difficult to predict the prognosis of this complication or the survival of these patients. Therefore, by following up on these patients in terms of survival rates and their association with the aforementioned factors, results can be reached that serve subsequent research related to diagnostic and therapeutic methods and the expected prognosis of this disease group.

Copyright: (C) 2021 the Author(s). This article is an open access article distributed under the terms and conditions of the Creative Commons Attribution (CC-BY) 4.0 license (https://creativecommons.org/licenses/by/4.0/). Published by Al-Kindi Centre for Research and Development, London, United Kingdom. 


\section{Methodology}

We conducted a study on patients with malignant and semi-malignant pleural effusions from one oncology center during the period between the beginning of January 2020 and the end of June 2020 and then followed up on each of them for a period of one year. The patient's gender and age at the time of MPE diagnosis were recorded. The primary tumor and its histological pattern were also identified. In addition, patients were classified according to whether they had undergone thoracentesis or biopsy, the results of the cytological study of pleural fluid, if performed, and the side of the effusion. We relied on Light's Criteria to diagnose exudative pleural effusions. The effusion was malignant when the cytology was positive, while we adopted the term "semimalignant' for effusions where the cytological study did not show malignant cells. Finally, we recorded whether or not patients underwent pleurodesis. Bleomycin was the only available material for pleurodesis on our hands at the time of the study. The survival time (ST) was calculated from the diagnosis of MPE until death or the passing of a year; whichever happens first. The final patient population was 89 .

This is an observational, prospective study. Statistical analysis methods include descriptive statistics which consist of qualitative variables expressed as frequencies and percentages, and quantitative variables expressed by measures of central tendency and measures of dispersion. Inferential statistics include survival time analysis using Kaplan-Meier curves, We used Cox regression analysis to estimate the Hazard Ratio and the Log-rank test to compare between groups and test the primitive variables. The results are considered statistically significant when the P-value is less than $5 \%$. We relied upon the IBM SPSS Statistics (Statistical Package for the Social Sciences for Windows; Version 20) program to calculate the statistical parameters and analyze the results.

\section{Results and Discussion}

The research sample included 89 patients divided into 53 men and 36 women. The ages ranged between 8 and 88 years. The median age was 58 years, while the mean age was 57. The sample was divided into two age groups: younger than 60 years old, and 60 years old or older. Investigation of pleural effusion was conducted in 59 patients, while the imaging findings were relied upon as the sole diagnostic tool in 30 patients. Thoracentesis was the diagnostic method of choice, and in the event that it was negative, a pleural biopsy was used. These 59 patients were then categorized into 35 cytology positive patients; when abnormal cells were found; and 24 cytology negative patients when no such cells were found. The patient sample was also divided into nine groups according to the primary tumor (Figure 1). Lung tumors were the most common with 25 patients, followed by hematologic malignancies and metastases of unknown origin. Moreover, patients made 12 groups according to their histological type (Figure 2). Adenocarcinoma was the most common with 25 patients, followed by non-Hodgkin's lymphoma and squamous cell carcinoma. The side of the effusion was verified in 66 out of 89 patients. The effusion was unilateral in 44 patients and bilateral in 22. Pleurodesis was performed in 21 patients (Table 1).

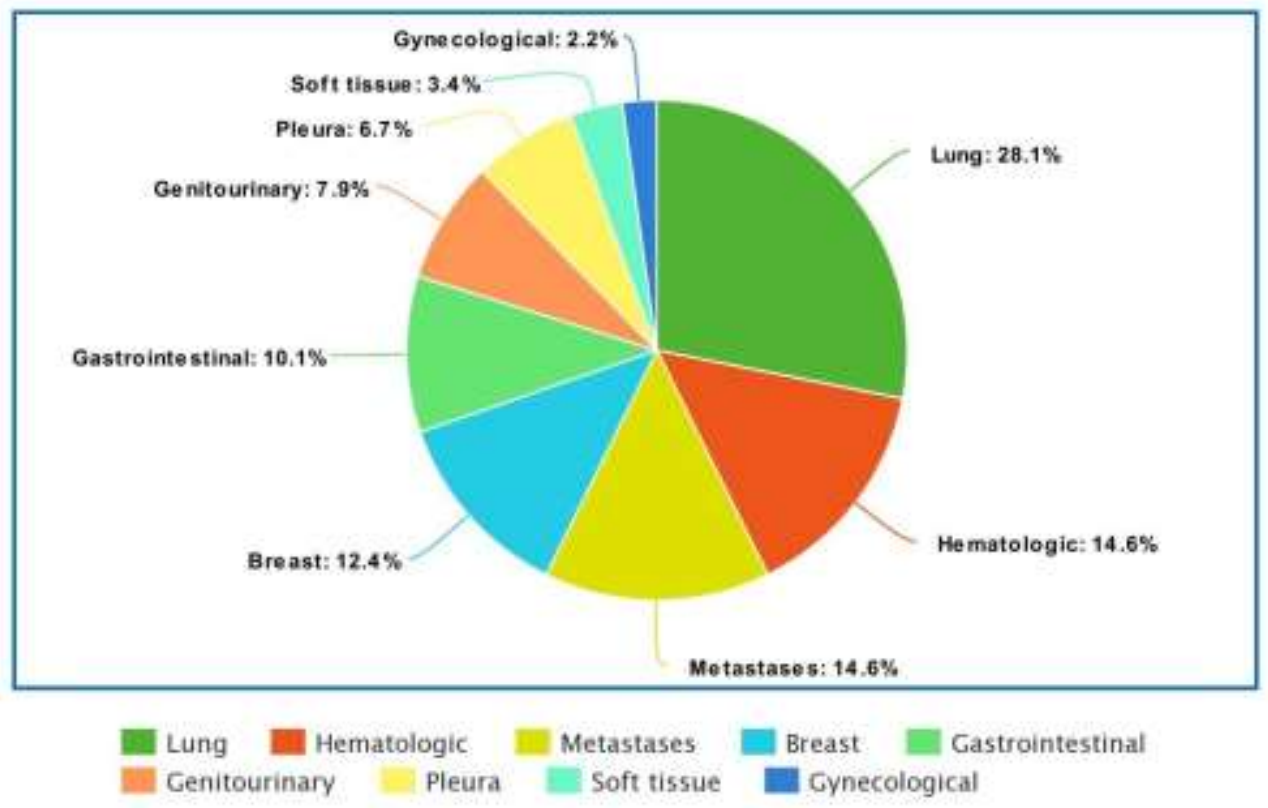

Figure 1. Pie chart demonstrating the distribution of the patient sample according to the primary tumor 


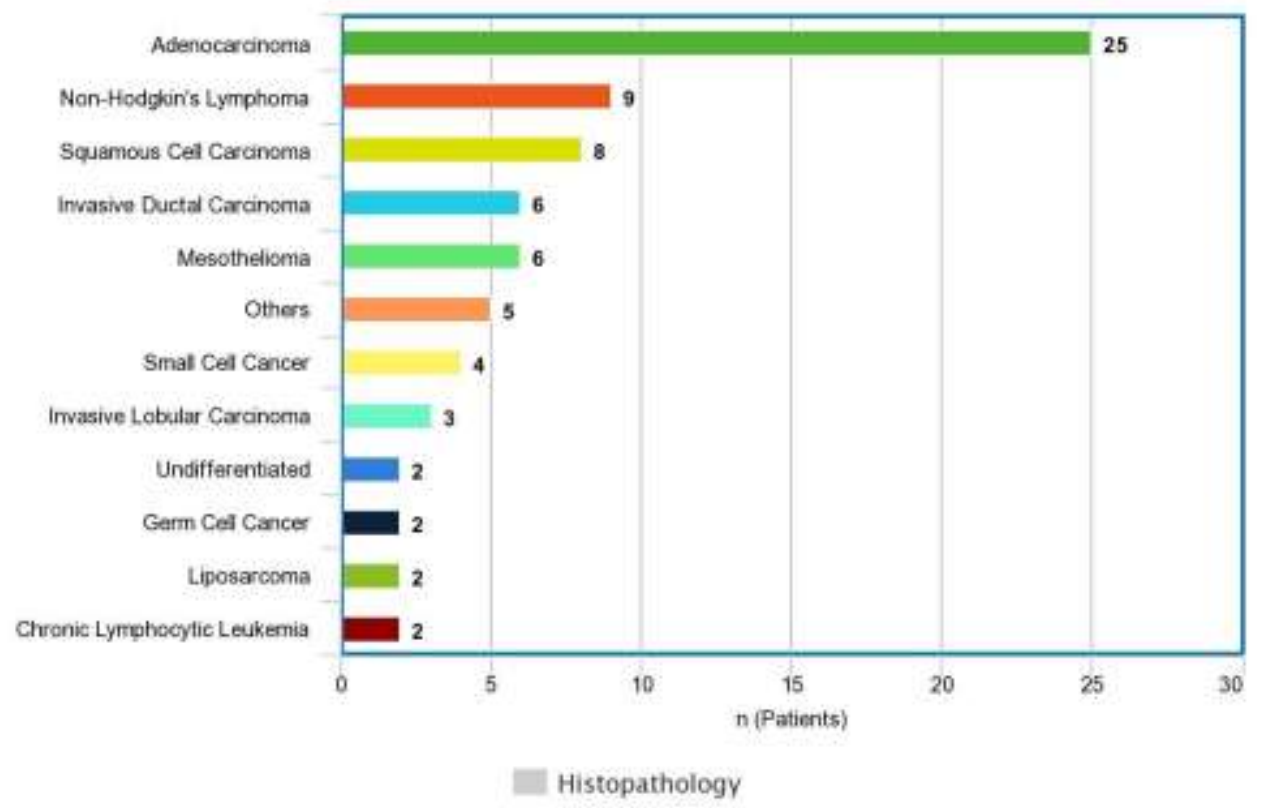

Figure 2. Bar chart demonstrating the distribution of the patient sample according to the histological type

Table 1. Characteristics of the study group

\begin{tabular}{|c|c|c|}
\hline Variable & Number (patients) & Percentage (\%) \\
\hline \multicolumn{3}{|l|}{ Gender } \\
\hline Male & 53 & 59.60 \\
\hline Female & 36 & 40.40 \\
\hline \multicolumn{3}{|l|}{ Age } \\
\hline$<60$ & 49 & 55.1 \\
\hline $60 \leq$ & 40 & 44.9 \\
\hline \multicolumn{3}{|c|}{ Tool of investigation } \\
\hline Thoracentesis & 38 & 42.7 \\
\hline Biopsy & 21 & 23.6 \\
\hline Imaging only & 30 & 33.7 \\
\hline \multicolumn{3}{|l|}{ Cytology } \\
\hline Positive & 35 & 59.3 \\
\hline Negative & 24 & 40.7 \\
\hline \multicolumn{3}{|l|}{ Side of effusion } \\
\hline Unilateral & 44 & 67 \\
\hline Bilateral & 22 & 33 \\
\hline \multicolumn{3}{|l|}{ Pleurodesis } \\
\hline Yes & 21 & 24 \\
\hline No & 68 & 76 \\
\hline
\end{tabular}


The mean survival time for the whole sample was 4.6 months, and the median survival time was 3 months. At the end of the oneyear follow-up period, only ten patients were alive. There was no statistically significant effect of gender or age on ST. The median survival time was higher in men without any difference in the mean. As for age, there was no difference recorded in the median or mean ST. There was also no statistically significant effect for the result of the cytological study on the ST survival time; with a clinical difference in favor of the negative cytology, up to two months for the median ST, and about one month for the mean ST. In a similar fashion, there was no statistically significant effect of lung and breast tumors on survival rates when compared with the rest of the primary tumors, although the P-value for breast tumors is close to a statistically significant value. Adenocarcinoma had a clinically significant effect on survival rates (Figure 3, Figure 4), and this statistical significance is associated with clinical significance observed through both the mean and the median ST; compared to the rest of the histological types. The side of effusion had no significant effect on survival rates. Finally, pleurodesis had a statistically significant effect on survival (Figure 5, Figure 6), as both the median and mean survival times were significantly higher in patients who had pleurodesis compared to those who did not undergo this procedure (Table 2).

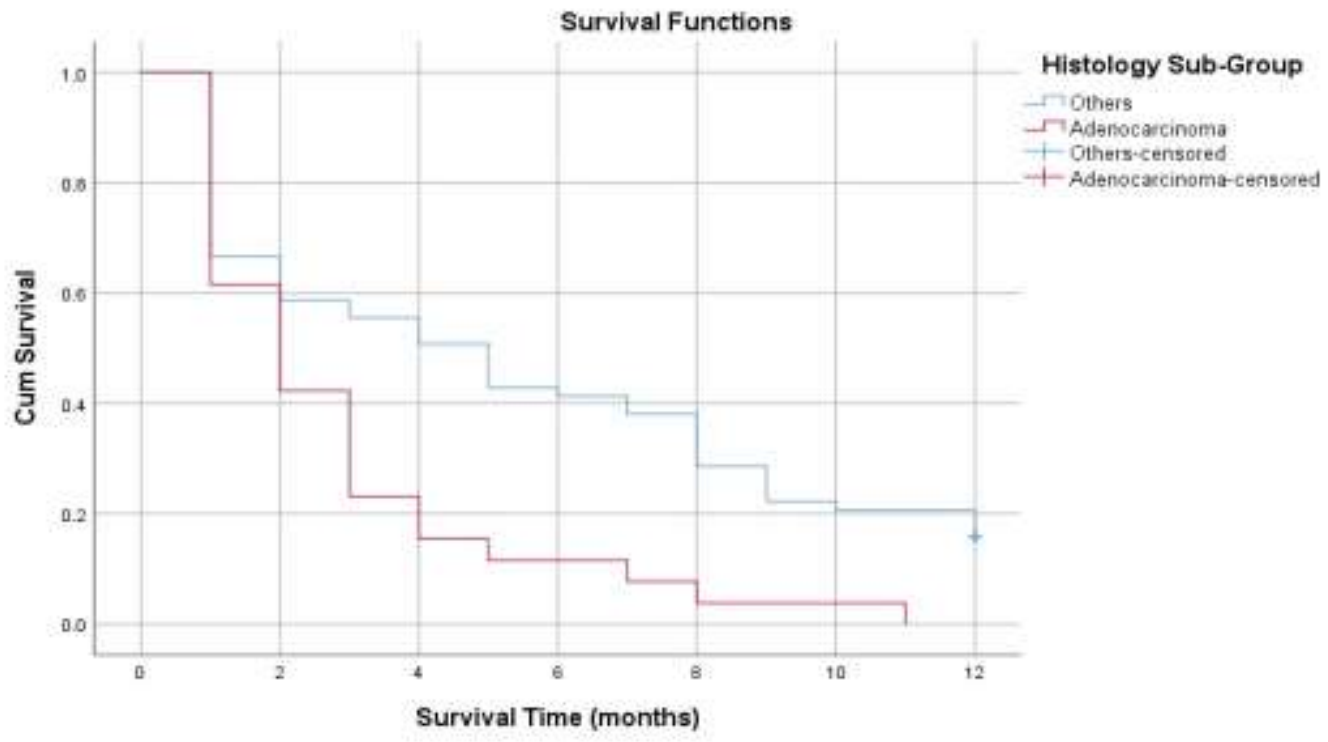

Figure 3. Kaplan-Meier survival curves according to histological type, showing a divergence between the curve of the adenocarcinoma group and the rest of the histological types, with an important difference between them in terms of survival time

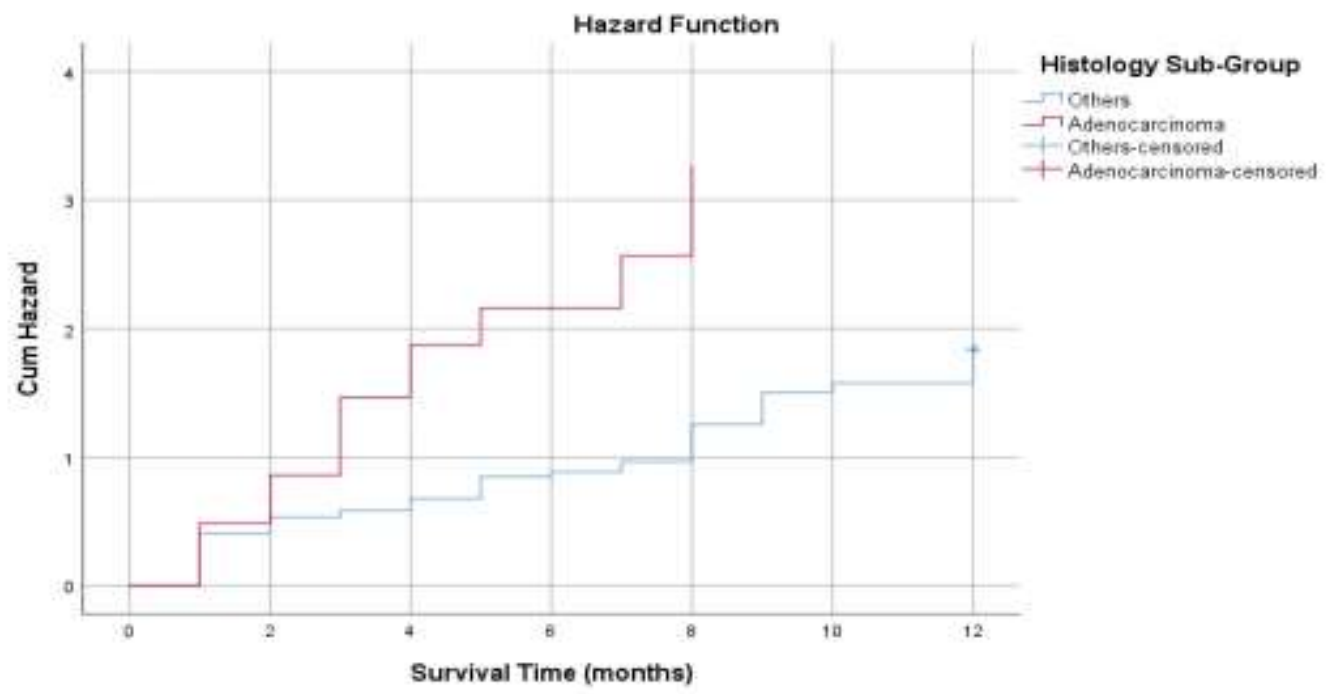

Figure 4. Kaplan-Meier risk curves according to histological type, showing a divergence between the curve of the adenocarcinoma group and the rest of the tumors, with an important difference between them in terms of survival time 


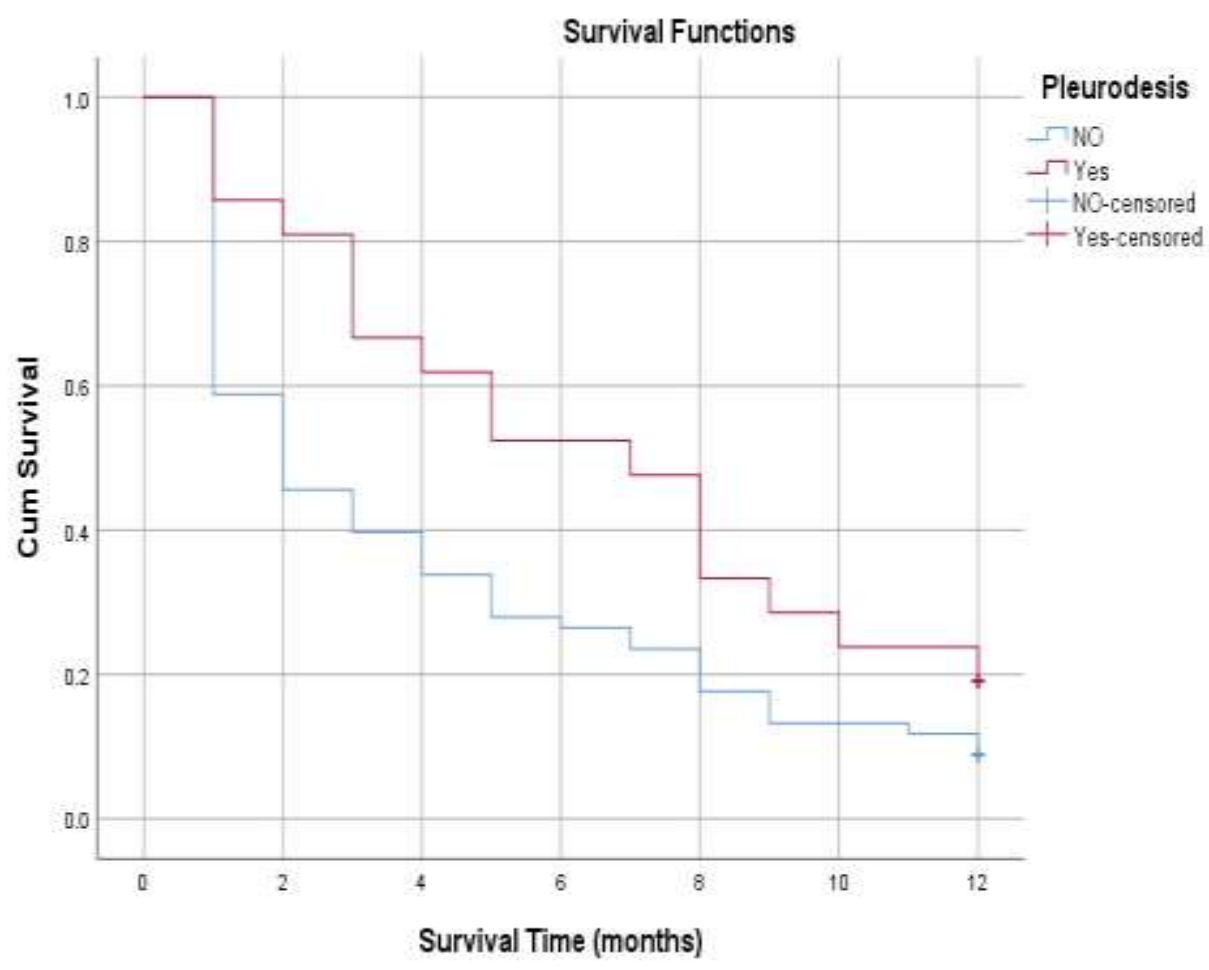

Figure 5. Kaplan-Meier survival curves according to whether or not pleurodesis was done; show a divergence between the two curves with an important difference between them in terms of survival time

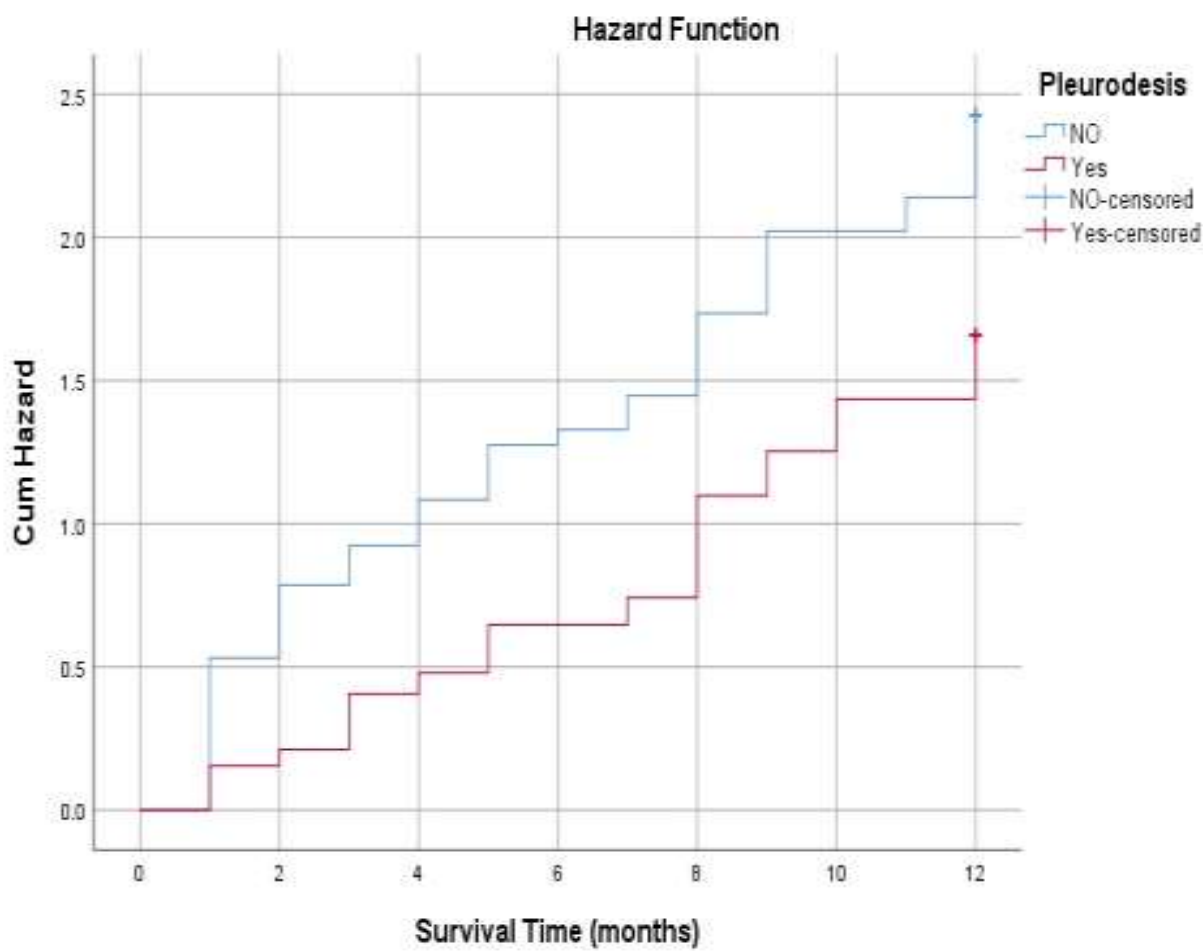

Figure 6. Kaplan-Meier risk curves according to whether or not pleurodesis was done; show a divergence between the two curves with an important difference between them I term of survival time 
Table 2. Mean and median survival according to each variable

\begin{tabular}{|c|c|c|c|c|}
\hline Variable & Number (patients) & P-value & Mean ST (months) & Median ST (months) \\
\hline \multicolumn{5}{|l|}{ Gender } \\
\hline Male & 53 & & 4.6 & 3 \\
\hline Female & 36 & 0.6 & 4.6 & 2.5 \\
\hline \multicolumn{5}{|l|}{ Age } \\
\hline$<60$ & 49 & & 4.7 & 3 \\
\hline $60 \leq$ & 40 & 0.8 & 4.5 & 3 \\
\hline \multicolumn{5}{|l|}{ Cytology } \\
\hline Positive & 35 & & 5.3 & 4 \\
\hline Negative & 24 & 0.4 & 6.2 & 6 \\
\hline \multicolumn{5}{|l|}{ Primary tumor } \\
\hline Lung & 25 & 0.4 & 4.9 & 3 \\
\hline Breast & 11 & 0.07 & 6.1 & 4 \\
\hline \multicolumn{5}{|l|}{ Histological type } \\
\hline Adenocarcinoma & 25 & 0.001 & 2.8 & 2 \\
\hline \multicolumn{5}{|l|}{ Side of effusion } \\
\hline Unilateral & 44 & & 4.2 & 3 \\
\hline Bilateral & 22 & 0.7 & 4 & 2.5 \\
\hline \multicolumn{5}{|l|}{ Pleurodesis } \\
\hline Yes & 21 & & 6.5 & 7 \\
\hline No & 68 & 0.04 & 4.1 & 2 \\
\hline
\end{tabular}

Malignant pleural effusion is associated with advanced tumor stages and poor patient survival (Heffner et al., 2003). No significant differences were recorded between our study and the studies conducted by Anevlavis et al. (2014) and Bikmalla et al. (2013) regarding the prevalence of malignant pleural effusion between genders, with men being the largest proportion in most studies, including ours, but the average age of patients at diagnosis was higher in these studies than ours. That may indicate an important point which is the increase in the incidence of MPE at younger ages in our country in particular. International studies varied in their method of diagnosing MPE. On the one hand, Bikmalla et al. (2013) resorted to thoracentesis as the most common diagnostic method, similarly to our study. On the other hand, Anevlavis et al. (2014) resorted to more invasive procedures such as thoracoscopy. According to Bikmalla et al. (2013), the cytological study was more commonly positive than our study in detecting malignant cells, and this may be due to the routine use of cell blocks, which are left in our hospital for special cases and are not carried out as usual. The use of imaging findings as to the only diagnosis of malignant pleural effusion - without conducting a cytological investigation - was less common than our study (Bikmalla et al., 2013). Lung tumor was dominant in both studies carried out by Bikmalla et al. (2013) and Jeba et al. (2018), which is consistent with our study. Regarding prevalence, lung tumors were followed by a wide variety of other tumors, without taking a specific trend (Bikmalla et al., 2013; Jeba et. al., 2018). The tendency of some tumors without others - or in greater proportions - to form MPEs can be explained by the presence of other proposed mechanisms accompanying the main mechanism for the occurrence of effusion. Antony et al. (2001) suggested a faulty cycle of interactions between pleural neoplastic cells and both the immune and vascular systems; leading to an increased fluid production through plasma leakage into the pleura. Stathopoulos \& Kalomenidis (2012) attributed the ability of some tumor cells to activate this sequence to a special and distinct transcriptional reservoir, which leads to active vascular interactions within the pleural space, a mechanism that also explains the tendency of some histological types to produce more MPEs than others. Explanatory adenocarcinoma was the most common histological type in both our study and Jeba et al (2018) study. Our study also agreed with Bikmalla et al. (2013) on the prevalence of unilateral effusion in patients with malignancy. Finally, the rates of pleurodesis were noticeably higher in international studies than in ours (Bikmalla et al., 2013; Hirata et al., 2011; Özyurtkan et al., 2010), and this disparity can be attributed to the different indications approved for pleurodesis between one hospital and another, 
and the high cost of this procedure in our country, which leads doctors to resort to less expensive and non-invasive procedures such as repeated thoracentesis. Gender or age did not affect survival rates according to Jeba et al. ${ }^{11}$, which is consistent with our study. Some international studies have found that some primary tumors have a significant statistical effect on survival rates (Anevlavis et al., 2014; Hirata et al., 2011; Jeba et al., 2018), which our study did not find mainly due to the small sample size. The results of international studies varied about the importance of the effect of histological type on survival rates. However, we found a statistical and a clinical value for the influence of adenocarcinoma on survival, a result that Jeba et al. (2018) couldn't find. The influence of the side of the pleural effusion has not been studied extensively in international studies, but Jeba et al. (2018) found that bilateral effusion is associated with lower survival rates, a result which our study could not prove. This may be due to our inability to determine the side of effusion in a significant part of the studied sample due to the lack of available data, which may have had a significant impact on our conclusion. As for pleurodesis; both Bikmalla et al. (2013) and Özyurtkan et al. (2010) focused on the safety of the procedure and its effectiveness in preventing the fluid from accumulating again, ideas that we could not address in our study. However, neither Bikmalla et al. (2013) nor Hirata et al. (2011) found statistical significance for the effect of pleurodesis on survival, contrary to our study which found an important effect of pleurodesis in favor of improving survival rates. This may be due to several reasons: the first is the high rates of pleurodesis globally compared to our study (Bikmalla et al., 2013; Hirata et al., 2011; Özyurtkan et al., 2010), then the variation in the indications for pleurodesis on which it depends, and finally the difference in the materials used in pleurodesis between studies. Of course, it should be noted that recent evidence indicates the superiority of talc over bleomycin - which is considered chemotherapy in itself for a large number of tumors such as lymphomas, testicular cancer, and others - in terms of success rates of pleurodesis and prevention of recurrence of effusion. Although there was no significant difference between them in mortality rates according to Wei et al. (2009).

\section{Conclusion}

We can conclude that MPE is associated with low survival rates of less than a year in most patients. The prevalence of this effusion does not differ significantly between genders or between age groups. Neither of these variables significantly affected survival rates. Lung tumors are the most common cause of MPE, followed by hematological malignancies and metastases of unknown origin. No primary tumor, including lung tumor and breast tumor, was associated with a statistically significant effect on survival rates. Adenocarcinoma is the most common histological type, and it is of great statistical importance in decreasing the survival rates of MPE patients. No significant differences were recorded in terms of frequency or survival rates between unilateral and bilateral effusions, or between negative and positive cytology. Finally, pleurodesis was associated with significantly better survival rates, both clinically and statistically.

To our knowledge, this is the first study that analyses the characteristics of MPE patients in our country. However, our study has its limitations. We could not evaluate the effectiveness of pleurodesis due to the difficulty of continuous, radiological follow-up of patients during the study period. The small size of patient groups according to tumor type and histological pattern negatively affected the strength of our findings and forced us to regroup patients in order to study the relationship of these two variables with survival rates. We also had to rely on imaging findings alone as a diagnosis of MPE in a group of sample patients. Plus, we were unable to determine the side of effusion in another group also due to the lack of available data.

Any pleural effusion that occurs in a patient with known malignancy should be investigated. The reliance on cell block technology should be widened to increase the sensitivity and specificity of screening for malignant cells. More extensive studies on the relationship of MPE with various types of tumors and their histological patterns should be conducted, and the impact of this relationship on survival rates must be studied in a wider aspect. We should also be giving special importance to adenocarcinoma patients who develop MPE because this effusion is associated with a significant decrease in survival rates. To conclude, pleurodesis should not be neglected because it is accompanied by a significant improvement in survival rates. Having better communication with thoracic surgeons about the possibility of updating or expanding the approved indications for performing pleurodesis could be of great importance, too.

Funding: This research received no external funding.

Acknowledgments: The authors would like to thank Dr. Mohammad Ramadan and Alissar Hejazeyah for their help with the statistical analysis.

Conflicts of Interest: The authors declare no conflict of interest.

\section{References}


[1] Anderson, C. B., Philpott, G. W., \& Ferguson, T. B. (1974). The treatment of malignant pleural effusions. Cancer, 33(4), 916-922. https://doi.org/10.1002/1097-0142(197404)33:4<916::AID-CNCR2820330405>3.0.CO;2-U

[2] Anevlavis, S., Kouliatsis, G., Sotiriou, I., Koukourakis, M. I., Archontogeorgis, K., Karpathiou, G., Giatromanolaki, A., \& Froudarakis, M. E. (2014). Prognostic Factors in Patients Presenting with Pleural Effusion Revealing Malignancy. Respiration, 87(4), 311-316. https://doi.org/10.1159/000356764

[3] Antony, V., Loddenkemper, R., Astoul, P., Boutin, C., Goldstraw, P., Hott, J., Panadero, F. R., \& Sahn, S. (2001). Management of malignant pleural effusions. European Respiratory Journal, 18(2), 402-419. https://doi.org/10.1183/09031936.01.00225601

[4] Asakura, H., Togami, T., Mitani, M., Takashima, H., Yokoe, K., Yamamoto, Y., Nishiyama, Y., Monden, T., Toyama, Y., \& Ohkawa, M. (2005). The usefulness of FDG-PET imaging for the radiotherapy treatment planning of pyothorax-associated lymphoma. Annals of Nuclear Medicine, 19(8), 725-728. https://doi.org/10.1007/bf02985123

[5] Bikmalla, S., Khalil, M., Ganaie, M., \& Haris, M. (2013). Characteristics of malignant pleural effusions. Review of one-year data from a large tertiary center. European Respiratory Journal, 42(Suppl 57). https://erj.ersjournals.com/content/42/Suppl_57/P2931

[6] Heffner, J. E., Highland, K., \& Brown, L. K. (2003). A Meta-analysis Derivation of Continuous Likelihood Ratios for Diagnosing Pleural Fluid Exudates. American Journal of Respiratory and Critical Care Medicine, 167(12), 1591-1599. https://doi.org/10.1164/rccm.200301-048pp

[7] Heffner, J. E., Nietert, P. J., \& Barbieri, C. (2000). Pleural Fluid pH as a Predictor of Survival for Patients With Malignant Pleural Effusions. Chest, 117(1), 79-86. https://doi.org/10.1378/chest.117.1.79

[8] Henschke, C. I., Yankelevitz, D. F., \& Davis, S. D. (1991). Pleural diseases: Multimodality imaging and clinical management. Current Problems in Diagnostic Radiology, 20(5), 157-181. https://doi.org/10.1016/0363-0188(91)90021-s

[9] Hirata, T., Yonemori, K., Hirakawa, A., Shimizu, C., Tamura, K., Ando, M., Katsumata, N., Tanimoto, M., \& Fujiwara, Y. (2011). Efficacy of pleurodesis for malignant pleural effusions in breast cancer patients. European Respiratory Journal, 38(6), 1425-1430. https://doi.org/10.1183/09031936.00171610

[10] Jeba, J., Cherian, R., Thangakunam, B., George, R., \& Visalakshi, J. (2018). Prognostic factors of malignant pleural effusion among palliative care outpatients: A retrospective study. Indian Journal of Palliative Care, 24(2), 184. https://doi.org/10.4103/ijpc.ijpc_183_17

[11] Özyurtkan, M. O., Balcı, A. E., \& Çakmak, M. (2010). Predictors of mortality within three months in the patients with malignant pleural effusion. European Journal of Internal Medicine, 21(1), 30-34. https://doi.org/10.1016/j.ejim.2009.09.012

[12] Prakash, U. B., \& Reiman, H. M. (1985). Comparison of Needle Biopsy With Cytologic Analysis for the Evaluation of Pleural Effusion: Analysis of 414 Cases. Mayo Clinic Proceedings, 60(3), 158-164. https://doi.org/10.1016/s0025-6196(12)60212-2

[13] Rice, T. W., Rodriguez, R. M., Barnette, R., \& Light, R. W. (2006). Prevalence and characteristics of pleural effusions in superior vena cava syndrome. Respirology, 11(3), 299-305. https://doi.org/10.1111/j.1440-1843.2006.00841.x

[14] Sahn, S. A. (1988). The Pleura. American Review of Respiratory Disease, 138(1), 184-234. https://doi.org/10.1164/ajrccm/138.1.184

[15] Stathopoulos, G. T., \& Kalomenidis, I. (2012). Malignant Pleural Effusion. American Journal of Respiratory and Critical Care Medicine, 186(6), 487-492. https://doi.org/10.1164/rccm.201203-0465pp

[16] Wei, Y., Yu, Q., \& Luo, H. (2009). Zhongguo Fei ai Za Zhi. Chinese journal of lung cancer, 12(3), 222-226. https://doi.org/10.3779/j.issn.10093419.2009.03.005 